\title{
Balancing Electricity Consumption and User Comfort in Load Management of Electric Tank Water Heaters
}

\author{
Alexander Belov \\ Pervasive Systems Research Group \\ University of Twente \\ Enschede, The Netherlands \\ a.belov@utwente.nl \\ Nirvana Meratnia \\ Pervasive Systems Research Group \\ University of Twente \\ Enschede, The Netherlands \\ n.meratnia@utwente.nl
}

\author{
Vadim Kartak \\ Department of Computer Science and Robotics \\ Ufa State Aviation Technical University \\ Ufa, Russia \\ kvmail@mail.ru \\ Paul J. M. Havinga \\ Pervasive Systems Research Group \\ University of Twente \\ Enschede, The Netherlands \\ p.j.m.havinga@utwente.nl
}

\begin{abstract}
In load management programs, electricity providers can reward their customer customers financially in return for the gained flexibility to reduce their loads at certain times on demand. In particular in residential sector, the question of whether or not to modify the energy consumption profile and how is highly challenging for an end-consumer due to difficulties in estimating the impacts on comfort of occupants. Therefore, it is essential for consumers to have means for estimation of the potential impact of load reduction on their comfort before the load reduction. We previously proposed an energy-comfort balancing model for electric tank water heaters (WHs) that can be used by residents upfront load reduction to estimate and fairly align their energy consumption for water heating to the desired level of thermal comfort. Unfortunately, that model provided solutions that were only nearly optimal. The model operated on a discrete temperature scale and thus suffered from rounding errors that accumulated with time. This paper presents a new model for energy-comfort balancing of WHs. Unlike the old model, the proposed model works on the temperature scale represented by an infinite set of real numbers. Thus, the model lacks errors resulting from the temperature discretization. The simulation results show that the refined model allows for higher energy savings due to the reduced stand-by heat losses of the WH as compared to the old model.
\end{abstract}

Keywords-water heaters, electrical load management, control algorithms, optimization, energy savings, user comfort

\section{INTRODUCTION}

The growing trends of residential electricity consumption and further decentralization of energy generation come along with changes in the entire electricity supply-demand chain today in the EU [1]. While electricity producers intend to optimally utilize their locally generated energy and sell it at the highest price, consumers aim at shifting and/or reducing their energy usage in response to high electricity prices. Not only the principles of load shifting and load reduction can cut consumer monthly electricity bills, but can also contribute to the overall stability of the grid if exercised during the periods of high electricity loads when reliability of the system is jeoperdised [2].
Peak electricity loads can cause contingencies in the power system such as frequency drops or failure of certain elements of the grid (e.g., generators, transformers, lines, etc.) which may lead to overloads in other branches, voltage collapse and possible blackouts [3]. Contingencies are thus highly undesirable and require grid operators to timely resolve them. Possible solutions for preventing and withstanding high load contingencies include, amongst others, the controllable reduction of load (so-called "brownouts"). Although consumers expect to be able to receive certain amounts of power at their discretion anytime, high load contingencies are not happening often and can be predicted to some extent which lets utility companies timely alert their customers about the upcoming peak-load periods and request some load reduction [4].

To diminish stress on the grid, utility companies encourage consumers to actively exchange their flexibility for reducing the loads at certain times in return for financial rewards (e.g., electricity tariff discounts). Modern utility programs $[5,6]$ offer technical means that allow the customers to receive steering signals (e.g., prices, utility messages) and to respond to them accordingly. The customers who have enough flexibility to shift their loads to the off-peak periods or to lower energy usage can acknowledge the load reduction requests and benefit financially.

In this light, a regular electricity consumer has to make a decision about how much energy to reduce (or to shift) considering the risk for personal comfort deterioration. An ill-conceived load management can have dramatic implications on end-user comfort. Impossibility to operate domestic devices and the need to alter consumption patterns might significantly undermine consumers' satisfaction with an energy provider. For example, in case of electrical water heating loads, cut-off of power supply during the periods of hot water usage might result in cold tap water and the user thermal discomfort followed by complaints to the utility company. Participating in utility load reduction programs is not as simple as it sounds. Besides the difficulties of estimating potential impact of load reduction on personal 
comfort (putting a number on user comfort), the consumer also needs to decide about his readiness to sacrifice his comfort, for example, for the sake of the grid stability (e.g., on the level of microgrid), monetary profits or lower environmental footprints. Therefore, to support consumers in load reduction process, there is a need for solutions that allow for balancing energy loads and end-user comfort.

In our previous paper [7], we demonstrated how such balancing could be achieved on the level of a single domestic device, an electrical tank water heater (WH). We considered a scenario where the electricity consumption of the WH was scheduled along the day based on the input from an ideal forecast ${ }^{1}$ of hot water demand and user comfort requirements ${ }^{2}$ so that the daily energy consumption for water heating is minimized while minimizing user comfort disruptions. To plan the heating periods of the WH during a day, we applied an LPI-optimization approach together with (i) discrete time and (ii) discrete state models. However, the downside of such "discrete" scheduling reveals in its inability to maintain the exact tank water temperature that optimally satisfies user comfort requirements. Specifically, the time discretization (i) ran the WH into situations where the tank water is underheated or overheated due to the too the fixed time-step size. The state model (ii) was responsible for holding the possible states of the WH that represent tank water temperature values. Because of the one-to-one mapping between the states and temperature values, a set of possible temperature values $\mathrm{T}$ was finite and restricted by the total number of states.

Importantly, having a finite set of temperatures resulted in rounding errors that accumulated with progress of simulations and caused an error between the tank water temperatures found by optimization solver from the finite set and the optimal real temperatures. One may think of compensating this side-effect of discretization by employing more states. However, in that case the optimization model size can increases dramatically. For example, there have to be 751 states to cover the range of temperatures from $15^{\circ} \mathrm{C}$ to $90^{\circ} \mathrm{C}$ with accuracy of $0.1^{\circ} \mathrm{C}$. Moreover, increasing the number of state only makes the rounding error grow slower, but does not solve the problem in its root.

In this paper, we specifically tackle the problem of rounding errors originating from the discrete state model (ii) and propose a new way to treat the states of a WH. Similar to the old optimization model, we aim at finding the trade-off between the daily energy consumption for water heating and user comfort. As before, our first objective function $\mathrm{f} 1$ is to minimize the daily user thermal discomfort. Unlike the old model, we translate the objective of saving electrical energy into the objective of keeping the tank water temperatures as low as possible. That is to say, we minimize the average tank water temperature during a day by means of objective function $\mathrm{f} 2$. This objective has the equivalent meaning as minimization of energy consumption of the WH, since the lower electricity consumption means lower tank water temperatures and vice versa. The central goal is then to

\footnotetext{
${ }^{1}$ Ideal forecast represented by start/end times of hot water events and water flows in the system contains no errors

${ }^{2}$ Comfort requirements provided by a test user represent parameters of the user thermal comfort model [7]. They include the desired tap water temperature, time and personal tolerance to water temperature deviations given by a user.
}

simultaneously minimize two objective functions f1 and $\mathrm{f} 2$. By analogy with the old model [7], we solve this problem in a two-step process.

The simulation results for various water usage profiles show that the proposed model outperforms the old model in the amounts of energy savings due to the reduced stand-by heat losses of the WH.

The rest of the paper is organized as follows. In Section 2, we introduce our setup, and briefly describe the standard operation of the WH. Section 3 provides the background information on the existing "old" model and highlights the issues related to it. Our new optimization model is presented in Section 4. The simulation results, discussion and conclusion can be found in the following sections.

\section{SETUP}

A typical domestic hot water system consists of a water heating unit, a tap mixer and a cold water supply connected by a system of pipes, as shown in fig 1 .

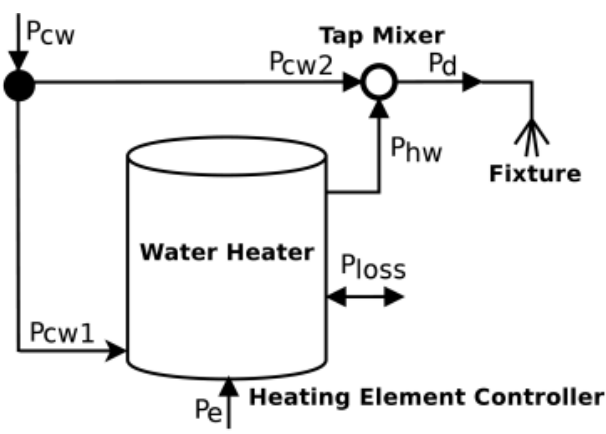

Fig. 1. Typical domestic hot water system

Domestic electric tank water heaters (WHs) are heating units that use water storage tanks to maximize their heating efficiency. The water inside the water reservoir is heated by electric heating elements Pe. Hot water is released from the top of the water tank Phw under the mains pipe pressure when the hot water tap is open. At the same time, that cold water enters the tank through the inlet at the bottom Pcw1 so that the volume of water inside always remains the same. Usually, users do not request water at high temperatures directly from the $\mathrm{WH}$, but rather use a mixer tap to obtain the desired tap water temperature and flow. The WH tank dissipates the heat to the surroundings in the form of heat losses Ploss.

The energy conservation law for the WH can be written in a simplified form as:

$$
M C_{p} \frac{d T}{d t}=P_{c w 1}+P_{e}-P_{h w}-P_{l o s s},
$$

where $\mathrm{M}$ is the mass of water inside the tank; $\mathrm{Cp}$ stands for heat capacity coefficient of water; $\mathrm{T}$ denotes the temperature of water inside the tank.

By solving Equation 1 for $\mathrm{T}$, one can derive the following relationship:

$$
T\left(t_{i+1}\right)=\left(\frac{\alpha_{i}}{\beta_{i}}+T\left(t_{i}\right)\right) e^{\beta_{i} t}-\frac{\alpha_{i}}{\beta_{i}},
$$

where $\alpha \mathrm{i}$ and $\beta \mathrm{i}$ are functions dependent on the water flow through the tank and engineering parameters of the $\mathrm{WH}$. 
Most of the WHs operate in a cyclic manner, meaning that their heating elements are either switched off or turned on to the maximum power to maintain the tank water temperature within some temperature deadband determined by the lower and the upper thermostat setpoint temperatures. The heating elements are turned on, when the water temperature is below the lower setpoint temperature inside the tank. Once the water temperature hits the upper setpoint, the heating elements are shut off and the process repeats. Unlike instantaneous (tankless) water heaters, the heating capacities of WHs are typically insufficient to maintain a stable tank water temperature during hot water usage. Which means that the tank water temperature inevitably drops when the hot water tap is open.

For simplicity and without loss of generality, we hereinafter consider a single-person apartment equipped with the medium-size WH (in our case, $80 \mathrm{~L}$ ) that provides service to sequential water events (no parallel hot water usage).

\section{EXISTING ENERGY-COMFORT MODEL}

As it follows from the standard deadband operation of WHs, the heat is stored not optimally during the $\mathrm{WH}$ operation. Firstly, the thermostat setpoints $\left(60-70^{\circ} \mathrm{C}\right)$ are typically lower than the maximum safety allowed threshold $\left(90^{\circ} \mathrm{C}\right)$. These restrict the maximum amount of heat that can be accumulated in the tank, which also means the higher potential thermal discomfort for the user, especially during intense hot water drains. Secondly, the backup of heat in the tank is continuously wasted with the stand-by heat losses to environment. Indeed, the standard operation attempts to always restore the tank water temperature drops back to the setpoints keeping the water tank at high temperatures even when the hot water is not really used.

In our previous paper [7], we proposed an energycomfort balancing optimization model for balancing daily energy consumption of the WH with user comfort. The idea of energy-comfort balancing is to store at any time only the exact amount of heat in the WH that optimally satisfies the expected user comfort requests. The model allowed a user for searching the water heating schedule that minimized the daily energy consumption of the $\mathrm{WH}$ while minimizing user comfort disruptions. The water was heated in the tank upfront the hot water usage and only up to the temperature dictated by the user comfort needs in this model. Whereas the water was maintained at cooler temperatures outside the hot water usage periods (e.g., at night) than in case of the standard operation. As a result, the stand-by heat losses of the WH declined and some energy savings could be achieved, since

We formulated the optimization problem as two conflicting goals - minimizing user thermal comfort disruptions F1 and minimizing electricity consumption F2 of the WH - using an LPI-optimization approach. The first objective function F1 to be minimized represented a possible user thermal discomfort that (s)he can experience due to (a) inadequate reserve of heat in the tank, and (b) a new lower level of electrical energy consumption of the WH. We used a user thermal comfort model $\mathrm{D}$ in order to quantify user inconvenience from cold tap water in [7]. The comfort model prescribed a number to every expected hot water event provided that parameters of hot water events are known from an ideal forecast and user input. The second objective F2 represented a daily energy consumption of the WH to be minimized subject to a user-acceptable thermal discomfort (from F1) and some engineering constraints of the $\mathrm{WH}^{3}$. We solved the problem in two steps by first minimizing F1 and then by incorporating the found value Dmin as a constraint during minimization of $\mathrm{F} 2$ at the second step. The model returned a binary vector $\mathrm{x}$, where each element $\mathrm{xi}=\{0,1\}$ corresponded to the state of the heating elements of the WH at time step i, i.e. either to keep the WH on or to shut it off.

More specifically, the formulated problem was based upon two assumptions: (i) we utilized a discrete time model with a finite horizon equal to one day (ahead) and a fixed time step $\Delta \mathrm{t}$, and (ii) we assumed that the tank water temperature that described the state of our WH was represented by a finite set of states. Because the state model imposed a one-to-one mapping between the states and the tank water temperatures, a set of tank water temperatures $\mathrm{T}$ had to be also finite, although not discrete. We assumed that the operating temperatures of the $\mathrm{WH}$ could vary from $15^{\circ} \mathrm{C}$ to $90^{\circ} \mathrm{C}$ with a step of $0.1^{\circ} \mathrm{C}$.

These two aforementioned assumptions have a direct impact on the accuracy of control solutions. Indeed, the assumption of a discrete timestep with a fixed size (i) makes the decision variable $\mathrm{xi}$ hold during the entire time $\Delta \mathrm{t}$. In real-life scenarios of domestic hot water usage, the hot water events may occur very frequently so that there may happen multiple (or no) hot water drains during the step $i$ with duration $\Delta \mathrm{t}$. Thereby, powering the $\mathrm{WH}$ along the entire time $\Delta t$ might be less efficient than switching the heating elements in fractions of $\Delta t$ in terms of user thermal comfort. The same is true for the case when the optimization solver decided to keep the WH switched off during $\Delta \mathrm{t}$. Therefore, the step-size of the discrete time model (i) has to be ideally very small to capture even insignificant temperature deviations. The obvious con of choosing a small step $\Delta t$ is the rapid growth of the optimization model size and as a result longer timer needed for optimization convergence.

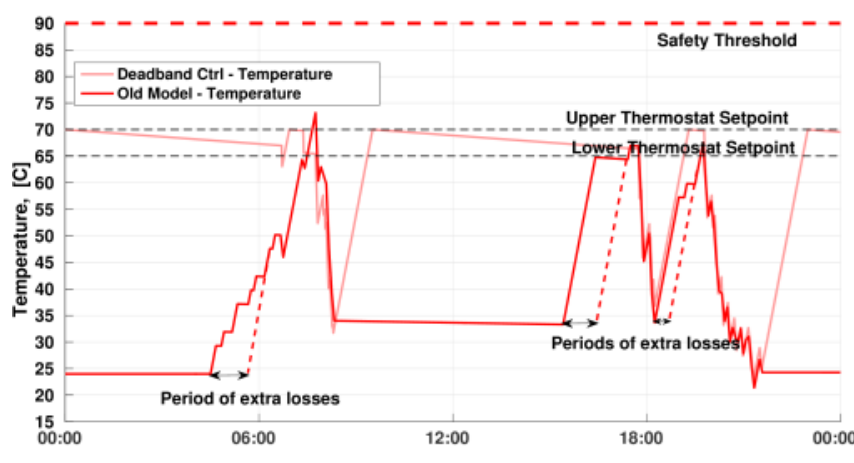

Fig. 2. Limitations of the old optimization model

On the other side, the assumption (ii) that manifests a finite set of states and tank water temperatures delivers rounding errors due to incapability of the optimization model to find temperatures with more than one decimal. To illustrate, in case the operational temperatures are in the range $[15: 0.1: 90]^{\circ} \mathrm{C}$ and the optimal tank water temperature at the end of step $\mathrm{i}$ is should be $50.15^{\circ} \mathrm{C}$, the absolute error at

\footnotetext{
${ }^{3}$ A lower energy consumption may be, for example, requested by a utility company or directly by a user.
} 
the end of step $\mathrm{i}$ will be $0.05^{\circ} \mathrm{C}$ either the solver comes up with $50.1^{\circ} \mathrm{C}$ or $50.2^{\circ} \mathrm{C}$. Importantly, the error of $0.05^{\circ} \mathrm{C}$ will affect the following interval $\mathrm{i}+1$ and lead to further error accumulation.

Another disadvantage that results from incapability of the old model to exactly maintain optimal tank water temperatures because of (i) and (ii) reveals in nearly optimally solutions obtained by the optimizer. Specifically, the optimization solver attempts to compensate errors of (i) and (ii) by interrupting the heating periods as illustrated in fig. 2. Although such interrupts make the temperature grow more accurately, i.e. this allows to heat the water up to the nearly optimal tank water temperatures, they introduce additional stand-by periods. Which means that the valuable heat is wasted even though there is no hot water usage. Therefore, it is more beneficial to heat the water exactly to the optimal temperature without breaking the heating periods into steps.

In this paper, we pursue the goal of downscaling the effect of rounding errors by modifying the assumption (ii) in our new model.

\section{PROPOSED MODEL}

One can notice that the graph of the function $T(t)$ in fig. 2 exhibits some linearities. The tank water temperature function $T(t)$ could thus be approximated by a linear piecewise function defined at each time slot $i$ on a discrete timescale as:

$$
y_{i}(t)=a_{i} t+b_{i}, \forall i \in[0, N], t \in[0, \Delta t] .
$$

After substituting the temperatures at the beginning and at the end of the interval $i T\left(t_{i}\right)$ and $T\left(t_{i+1}\right)$ in (3) and subtracting two equations we get:

$$
T\left(t_{i+1}\right)=T\left(t_{i}\right)+a_{i} \Delta t=T\left(t_{i}\right)+y_{i}{ }^{\prime}\left(t_{i}\right) \Delta t .
$$

We can further use Equation (4) for finding the future temperature values $T\left(t_{i+1}\right) \ldots T\left(t_{i+n}\right)$ by plugging the known temperature value $T\left(t_{i}\right)$, the time step size $\Delta t$ and the unknown value of the tangent $y_{i}{ }^{\prime}\left(t_{i}\right)$ to the approximation function function $y_{i}(t)$ at time $t_{i}$. In fact, it can be seen from fig.2 that the slope of the function $T(t)$ remains almost the same within the heating (or cooling) cycles. Taking into account that the incline of $T(t)$ becomes slightly flatter when heating the water in the range of high temperatures ${ }^{4}$, we divide the whole range of possible temperatures $\left[T_{c w}, T_{\max }\right]$ into $M$ number of even zones $\frac{T_{\max }-T_{c w}}{\Delta T}$. Therefore, temperature values $T\left(t_{i}\right)$ that belong to the same zone $j$ will have the same value of tangent value $a_{j}=y^{\prime}(t)$. Equation 3 can be then re-written as:

$$
T\left(t_{i+1}\right)=T\left(t_{i}\right)+a_{j} \Delta t=T\left(t_{i}\right)+y_{j}{ }^{\prime}\left(t_{i}\right) \Delta t, \forall j \in M .
$$

Each interval of temperatures $j$ represents a state of the WH. The benefit is that we let a fewer number of states cover infinite temperature ranges. The tank water temperature $T\left(t_{i+1}\right)$ at the end of the step $i$ of the discrete time model can take now any real value. The idea is to replace the old state model with a new state model where each state of the WH tank is represented by a range of temperatures rather than by a single temperature value and every temperature can be obtain by means of Equation 4'. We first compute all possible temperature transitions $\left\{T\left(t_{i}\right), T\left(t_{i+1}\right)\right\}_{j}$ for every time interval $i$ and every temperature zone $j$ by plugging the tank water temperatures at the beginning of each zone $T\left(t_{i}\right)$, the known time step size $\Delta t$ and parameters $\alpha_{i}$ and $\beta_{i}$ into Equation 2. The found values $T$ are used further to calculate tangents $a_{i j}$ by means of Equation 3' that can be arranged in matrices $\boldsymbol{A}+$ and $\boldsymbol{A}$ - of size $N \mathrm{x} M$, each corresponding to the cases when the WH is turned on and off, respectively. Secondly, we compute all thermal discomfort levels $D(i, j)$ that the user can potentially experience at any time step $i$ when the temperatures are $\left\{T\left(t_{i}\right), T\left(t_{i+1}\right)\right\}_{j}$ at the beginning and at the end of step $i$, respectively. The outcomes can be organized in matrices $\boldsymbol{D}+$ and $\boldsymbol{D}$ - representing the cases when the WH is turned on and off, respectively. Thus matrices $D+$ and $\boldsymbol{D}$ - establish the relations between the input temperatures $T_{j}\left(t_{i}\right)$ and the resulting discomfort values $D(i, j)$. Two available control alternatives for WH at every time step $i$ weather or not to heat the water - can be represented by an binary vector $\boldsymbol{x}, x_{i}=\{0,1\}$ of size N. Let us introduce two auxiliary binary matrices $\gamma+$ and $\gamma$ - responsible for the choice of the optimal transitions $\left\{T\left(t_{i}\right), T\left(t_{i+1}\right)\right\}_{j}$ and the optimal state of the WH $x_{i}=\{0,1\}$ at every step $i$.

The optimization problem for optimal scheduling of $\mathrm{WH}$ heating can be formulated as finding such temperatures $T\left(t_{i}\right)$ that optimally satisfy two objective functions, namely minimization of the daily electricity consumption of the WH and minimization of the user comfort disruptions. Let us $f_{1}$ denote the total daily discomfort and $f_{2}$ the daily energy consumption of the WH. The problem can be formalized as follows:

$$
\left\{\begin{array}{c}
\min \left[\sum_{i=1}^{N} \sum_{j=1}^{M} \gamma+_{i, j} D+_{i, j}+\gamma-_{i, j} D-_{i, j}\right] \\
\min \left[\sum_{i=1}^{N} T_{i}\right]
\end{array}\right.
$$

s.t.:

$$
\begin{gathered}
\sum_{j=1}^{M} \gamma+_{i, j}=x_{i} \\
\sum_{j=1}^{M} \gamma^{-}{ }_{i, j}=1-x_{i} \\
T_{i}=T_{i-1}+\Delta t\left[\sum_{j=1}^{M} \gamma_{i, j} A+_{i, j}+\gamma_{i, j} A-_{i, j}\right] \\
\sum_{j=1}^{M} \gamma_{i, j}(j-1) \leqslant T_{i-1} \\
\sum_{j=1}^{M} \gamma_{i, j}>T_{i-1} \\
T_{i}<T_{\max } \\
\forall i \in[1, N], j \in[1, M]
\end{gathered}
$$

4 Similarly the temperature $\mathrm{T}(\mathrm{t})$ drops slower in the range of colder temperatures when the $\mathrm{WH}$ cools down without hot water usage. 
Expression (5) represents the goal to minimize the total daily user thermal discomfort, while Equation (6) is responsible for the objective of minimization of the total daily energy consumption for heating.

A set of constraints (7a)-(7f) governs the optimization problem. Thus, constraints (7a) and (7b) together dictate that only one temperature transition $\left\{T\left(t_{i}\right), T\left(t_{i+1}\right)\right\}_{j}$ is possible at time step $i$ regardless to the state of the WH $\{$ on/off $\}$. The constraint $(7 \mathrm{c})$ represents the condition of the temperature transitions in our new state model (Equation 4') for every step $i$. At the same time, the constraints $(7 \mathrm{~d})$ and $(7 \mathrm{e})$ serve for finding the correct indices of the temperature transitions in matrices $\boldsymbol{A}+$ and $\boldsymbol{A}$-. Finally, the constraint (7f) tells that the maximum temperature inside the tank must not exceed the safety temperature threshold.

We solve the problem stated in (5)-(7) in two steps. At step \#1 we find the solution $\boldsymbol{x}^{*}$ (or vector of temperatures $\left.T^{*}\right)$ by minimizing the objective function $f_{1}$ in Equation (4) s.t. the constraints in (7). The obtained value $f_{1}^{*}=f_{1}\left(\mathrm{x}^{*}\right)$ represents the minimum possible discomfort value that could be theoretically achieved. At step \#2 we incorporate the found value $f_{1} *$ in the form of extra constraints $(7 \mathrm{~g})$ and $(7 \mathrm{~h})$ when minimizing the objective function $f_{2}$ in Equation (6) s.t. the constraints in (7):

$$
\begin{gathered}
\sum_{j=1}^{M} \gamma_{i, j} D+_{i, j}+\gamma_{i, j} D-_{i, j}>0 \\
\sum_{j=1}^{M} \gamma_{i, j} D+_{i, j}+\gamma_{i, j} D-_{i, j} \leqslant f_{1} *, \\
\forall i \in[1, N], j \in[1, M]
\end{gathered}
$$

\section{MODEl EVALUATION \& RESUlTS DISCUSSION}

\section{Simulation results}

This section presents the evaluation results of the new optimization model proposed in Section 4 compared to the old model discussed in Section 3 and the standard deadband control of the WH outlined in Section 2. We are specifically interested in how the new model that operates with real temperatures influences the heat losses and as a result the electricity consumption for water heating. We demonstrate the effect of the new model on the accuracy of the WH scheduling by comparing the electricity consumption achieved by means of each model. For this, we fix the user thermal comfort at the level experienced by the user during the standard (deadband) control of the $\mathrm{WH}$.

We validate the accuracy of the new model based on daily and weekly hot water consumption profiles in HWS shown in fig, 1, while upholding the assumptions about the setup and hot water usage discussed in Section 2 and Section 3 . The output of the optimization solver Gurobi, vectors of heating periods $\mathrm{x}$, are used as input for simulation algorithm of the WH in order to obtain actual results of the control.

The simulation results of the selected daily profile of hot water usage and associated user comfort preferences are illustrated in fig. 3 and fig. 4 . The simulations of the selected weekly hot water usage profile can be found in fig. 5 .

\section{Results Discussion}

The tank water temperature graph of the new model lacks the periods of extra heat waste as shown in fig. 3. The heating periods do not interrupt in the new model in order to fit the optimal tank water temperatures required at the beginning of hot water usage as in the old model. Therefore, no steps are created during the heating, meaning lesser heat losses. Because the new state model treats water temperatures as real (continuous) numbers from an infinite set, and because the objective to minimize the electricity consumption is translated into the objective of minimization of the daily average temperature, the Gurobi solver finds temperature values that are close to the actual values during simulations. The reduced heat losses in the new model yield additional energy savings at the same level of the user comfort in comparison to the old model.

As shown in fig. 4, Pareto front of solutions of the new model lays below the front of the old model. The increase in accuracy of the new temperature model results in additional energy savings of around $0.19 \mathrm{kWh}$ for a single working day as compared to the old model. These energy savings can be explained by mainly the lowered by $0.12 \mathrm{kWh}$ heat losses and slightly lower (by $0.07 \mathrm{kWh}$ ) hot water consumption from the tap at the same level of user comfort. The total energy savings achieved with the new model constitute 0.45 $\mathrm{kWh}$ per day as opposed to the standard operation. The total energy savings can be explained by the reduced by $0.43 \mathrm{kWh}$ heat losses and diminished by $0.02 \mathrm{kWh}$ hot water consumption from the tank. A slight decrease of the hot water consumption originates from the fact that tank water temperatures are higher in the new model, and thus less hot water is needed to provide the same level of comfort.

The weekly simulations of new model yield $4.44 \mathrm{kWh}$ of energy savings compared to the standard control as against $0.99 \mathrm{kWh}$ of energy savings in the old model. Energy savings achieved by the new model during the first 5 working days improved by $147 \%$ (from $1.33 \mathrm{kWh}$ to 3.30 $\mathrm{kWh}$ ) as opposed to the old one with average daily savings of $0.66 \mathrm{kWh}$. Moreover, the new model was capable to achieve $1.14 \mathrm{kWh}$ of energy savings during the weekend $(0.57 \mathrm{kWh}$ in average), whereas the old model demonstrated energy increase by $0.34 \mathrm{kWh}$ as compared to the standard control.

\section{CONCLUSION}

This paper presents a new model for optimal scheduling of water heating of domestic electrical tank water heaters (WHs) in terms of both the electrical energy consumption and user thermal comfort. The proposed optimization model benefits from the improved state model that treats tank water temperatures on a infinite set of real numbers. The model is evaluated based on simulations for the case of a singleperson apartment. The simulation results are put next to the results of the old model [7] and the standard deadband control of the WH.

The simulation results for a single working day show the advantage of the new optimization model over the old one in terms of additional energy savings due to the reduced heat losses. The new model outperforms the old model by around $73 \%$ (enhance by $0.19 \mathrm{kWh}$ ) of higher energy savings which correspond to the reduction of the total daily energy 
consumption by $2.3 \%$ as compared to the standard deadband control based on simulations for a single working day. The simulations based on a weekly profile indicate that the new model is capable to achieve by $2.07 \%$ greater energy savings than the old model.

\section{ACKNOWLEDGMENT}

We would like to express our gratitude to the Go-Green project funded by the Netherlands Enterprise Agency and to Russian Foundation of Basic Research (RFBR) for providing a research grant 19-07-00895-a.

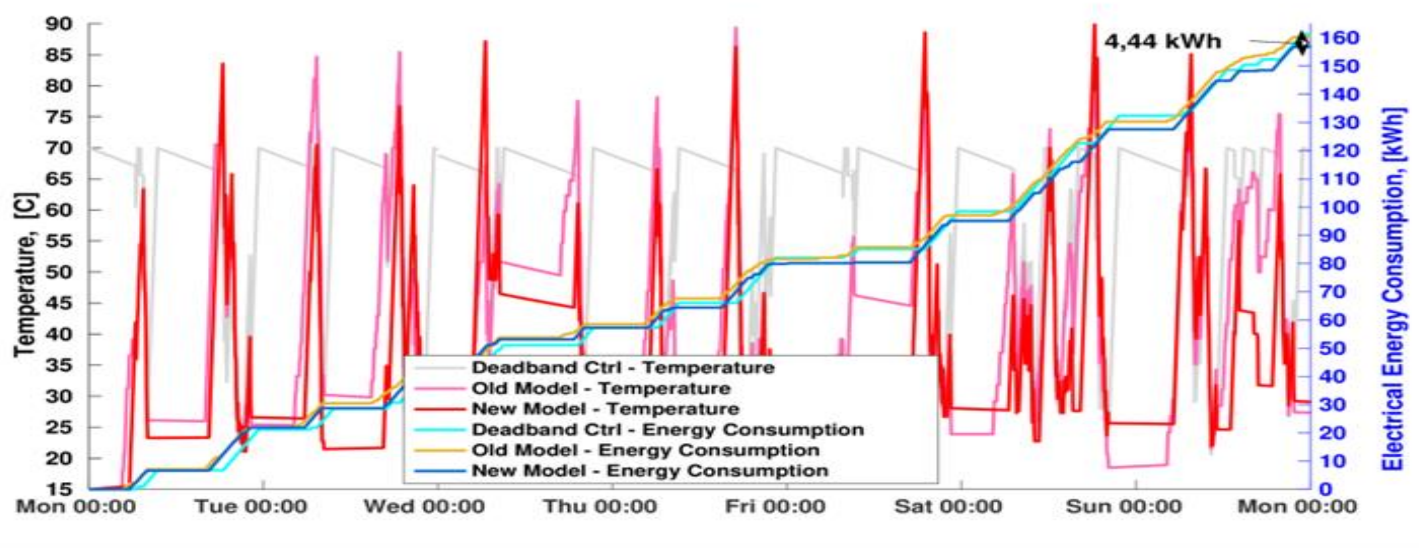

Fig. 3. Pareto front of solutions for a working day profile

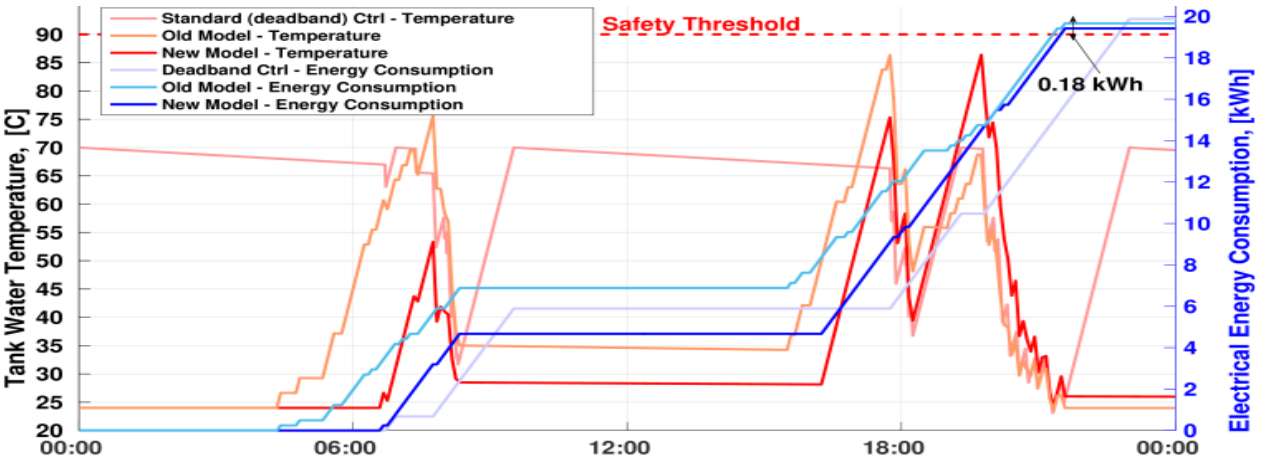

Fig. 4. Control for a working day profile effect on energy savings and comfort

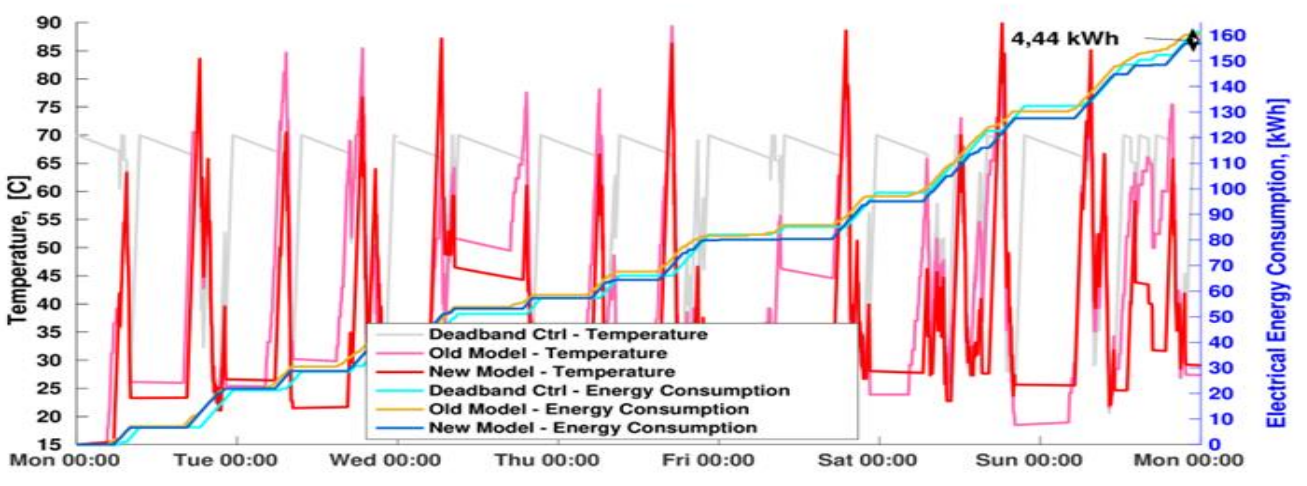

Fig. 5. Simulations based on a weekly profile 


\section{REFERENCES}

[1] Report "Final household electricity consumption by use". The European Environment Agency, the EU, 2016.

[2] Staff report "Assessment of demand response and advanced metering”. Federal Energy Regulatory Commission, USA, 2018

[3] J. Seymour, T. Horsley, "The seven types of power problems". APC, USA, 2005

[4] V. Giordano, F. Gangale F et al., "Smart Grid projects in Europe: Lessons learned and current developments". European Commission, Joint Research Centre, 2019.
[5] Power Manager project, https://www.dukeenergy.com/home/products/power-manager. Duke Energy, USA, last visit: 15-06-2019.

[6] Duurzame Opwek project, https://www.alliander.com/nl/content/duurzame-opwek. Alliander, The Netherlands, last visit: 15-06-2019.

[7] A. Belov, V. Kartak, A. Vasenev, N. Meratnia, and P. J. Havinga "A hierarchical scheme for balancing user comfort and electricity consumption of tank water heaters". In Proc. of 2016 IEEE Power \& Energy Society Innovative Smart Grid Technologies Conference (ISGT), USA, vol. Online ISSN IEEE Xplore $=2472-8152$. IEEE, 2016, pp. 1-5. 